\title{
Unexpected emergence from the vegetative state: delayed discovery rather than late recovery of consciousness
}

\author{
Willemijn S. van Erp ${ }^{1,2}\left(\mathbb{D} \cdot\right.$ Anoek M. L. Aben $^{1} \cdot$ Jan C. M. Lavrijsen ${ }^{1} \cdot$ Pieter E. Vos $^{3} \cdot$ Steven Laureys $^{2}$. \\ Raymond T. C. M. Koopmans ${ }^{1,4}$
}

Received: 28 April 2019 / Revised: 4 September 2019 / Accepted: 12 September 2019 / Published online: 20 September 2019

(c) The Author(s) 2019

\begin{abstract}
Background The vegetative state, also known as the unresponsive wakefulness syndrome, is one of the worst possible outcomes of acquired brain injury and confronts rehabilitation specialists with various challenges. Emergence to (minimal) consciousness is classically considered unlikely beyond 3-6 months after non-traumatic or 12 months after traumatic etiologies. A growing body of evidence suggests that these timeframes are too narrow, but evidence regarding chances of recovery is still limited.

Objective To identify the moment of recovery of consciousness in documented cases of late emergence from a vegetative state.

Methods Four cases of apparent late recovery of consciousness, identified within a prospective cohort study, were studied in-depth by analyzing medical, paramedical and nursing files and interviewing the patients' families about their account of the process of recovery.

Results All patients were found to have shown signs of consciousness well within the expected time frame ( 5 weeks -2 months post-ictus). These behaviors, however, went unnoticed or were misinterpreted, leading to a diagnostic delay of several months to over 5 years. Absence of appropriate diagnostics, the use of erroneous terminology, sedative medication but also patientrelated factors such as hydrocephalus, language barriers and performance fluctuations are hypothesized to have contributed to the delay.

Conclusions Delayed recognition of signs of consciousness in patients in a vegetative state may not only lead to suboptimal clinical care, but also to distorted prognostic figures. Discriminating late recovery from the delayed discovery of consciousness, therefore, is vital to both clinical practice and science.
\end{abstract}

Keywords Consciousness disorders $(\mathrm{MeSH}) \cdot$ Persistent vegetative state $(\mathrm{MeSH}) \cdot$ Neurological rehabilitation $(\mathrm{MeSH})$

Electronic supplementary material The online version of this article (https://doi.org/10.1007/s00415-019-09542-3) contains supplementary material, which is available to authorized users.

Willemijn S. van Erp

Willemijn.vanerp@radboudumc.nl

1 Department of Primary and Community Care, Radboud Institute for Health Sciences, Radboud University Medical Center, Radboudumc, ELG 117, Postbus 9101, 6500 HB Nijmegen, The Netherlands

2 Coma Science Group, GIGA Consciousness, Université de Liège, Liège, Belgium

3 Department of Neurology, Slingeland Ziekenhuis, Doetinchem, The Netherlands

4 Joachim en Anna, Center Specialized Geriatric Care, Nijmegen, The Netherlands

\section{Introduction}

The vegetative state, also known as the unresponsive wakefulness syndrome (VS/UWS) is one of the worst possible outcomes of acquired brain injury [1,2]. Patients show spontaneous eye opening but no behavioral signs of consciousness. Emergence from VS/UWS is classically considered unlikely beyond 3-6 months after non-traumatic or 12 months after traumatic etiologies [3]. These timeframes, however, are likely inaccurate $[4,5]$. The detection of even minimal awareness is of major clinical importance. In contrast to VS/UWS, patients in a minimally conscious state (MCS) [6] show residual sensory and emotional processing, including nociception $[7,8]$ and have better chances of further recovery [5]. Behaviorally, MCS is characterized 
by signs of awareness of the self and/ or the environment without functional communication or functional object use [6]. Based on the absence or presence of evidence for language processing, patients are considered respectively to be in MCS- (showing for example visual pursuit or localization of noxious stimuli) or in MCS+ (showing reactions such as inconsistent command following and intentional communication) [9].

Differentiating VS/UWS from MCS unfortunately remains challenging, as reflected by a high rate of misdiagnosis [10]. In this context of diagnostic and prognostic uncertainty, clinicians and families face vital decisions on treatment goals and limitations.

We present four VS/UWS patients in whom signs of consciousness were identified beyond the aforementioned timeframes and propose clinical and scientific recommendations that arise from their stories.

\section{Methods}

Patients were identified within a cohort of 31 patients in VS/ UWS at least 1 month post-ictus, founded in the Netherlands in 2012. On-site assessment of level of consciousness (LoC) by a trained clinical researcher ( $\mathrm{WvE})$ consisted, at baseline and at each follow-up, of a single Coma Recovery Scalerevised (CRS-R) [11] plus observation of possibly conscious behavior reported by proxies and staff. Findings, including the diagnostic uncertainty associated with this single assessment, were explicitly communicated with the treating physician. We invited families and staff to contact us between follow-up visits if the patient's reactions changed.

For this case series, we included patients with a CRS-R based VS/UWS diagnosis, in whom the first signs of consciousness were detected during formal follow-up at or after 6 (non-traumatic) or 12 months (traumatic brain injury). Patients' representatives gave written, informed consent. A medical research ethics committee concluded that no complementary review was needed.

Clinical files were searched for descriptions of possibly conscious behavior. We compared proxies' accounts of the course of recovery to the chronology of the formal diagnostic process. ${ }^{1}$

\footnotetext{
$\overline{1}$ Anonymized data will be shared by request from any qualified investigator.
}

\section{Results (see Table 1; full CRS-R scores available as supplementary material).}

\section{Patient 1}

A 49-year-old male, non-Dutch speaking, sustained hypoxic brain damage during an out-of-hospital cardiac arrest in 2013. With a diagnosis of 'coma vigil' he was discharged to a regular nursing home, where the physician described his condition as a 'comatose state'. After two research-related CRS-R assessments corresponding to a diagnosis of VS/ UWS, during the third examination, 8 months post-ictus, he showed automatic motor behavior. Medical files revealed that in the first 3 months after the incident, the patient manually removed his tracheal cannula twice and tracked visual stimuli with his eyes. The eventual diagnosis of MCS did not change the patient's treatment. Two years and 8 months post-ictus, he remains in MCS-, unable to communicate, immobile, and dependent on others for all activities of daily living.

\section{Patient 2}

A 64-year-old female suffered a subarachnoid hemorrhage in 2008 and was transferred to a specialized nursing home without formal LoC diagnosis seven weeks later. Upon admission, the physician concluded she was in a 'vegetative state', although she showed 'some visual tracking'. The patient received valproic acid for epilepsy and increasing doses of midazolam to treat spasticity. Four years and 9 months post-ictus valproic acid was discontinued. Within weeks, the patient's family noted her smiling in response to the names of loved ones. Her diagnosis did not change, however, until the clinical researcher $(\mathrm{WvE})$ identified visual pursuit and automatic motor behavior, corresponding to MCS at 5.5 years post-ictus. Midazolam was discontinued, but no interventions aimed at further recovery took place. At the time of this study, over 7 years post-ictus, the patient inconsistently follows commands (corresponding to MCS+) but is unable to communicate.

\section{Patient 3}

A 27-year-old male sustained extensive traumatic brain injury (diffuse axonal injury, acute subdural hemorrhage and traumatic subarachnoid hemorrhage) during a car accident in 2011. Without explicit LoC diagnosis, 5 weeks later he was admitted to a specialized nursing home with a therapy program aimed at recovery of consciousness. Despite the presence of visual pursuit and localization of noxious stimuli, his physician considered him to be in a 'vegetative 


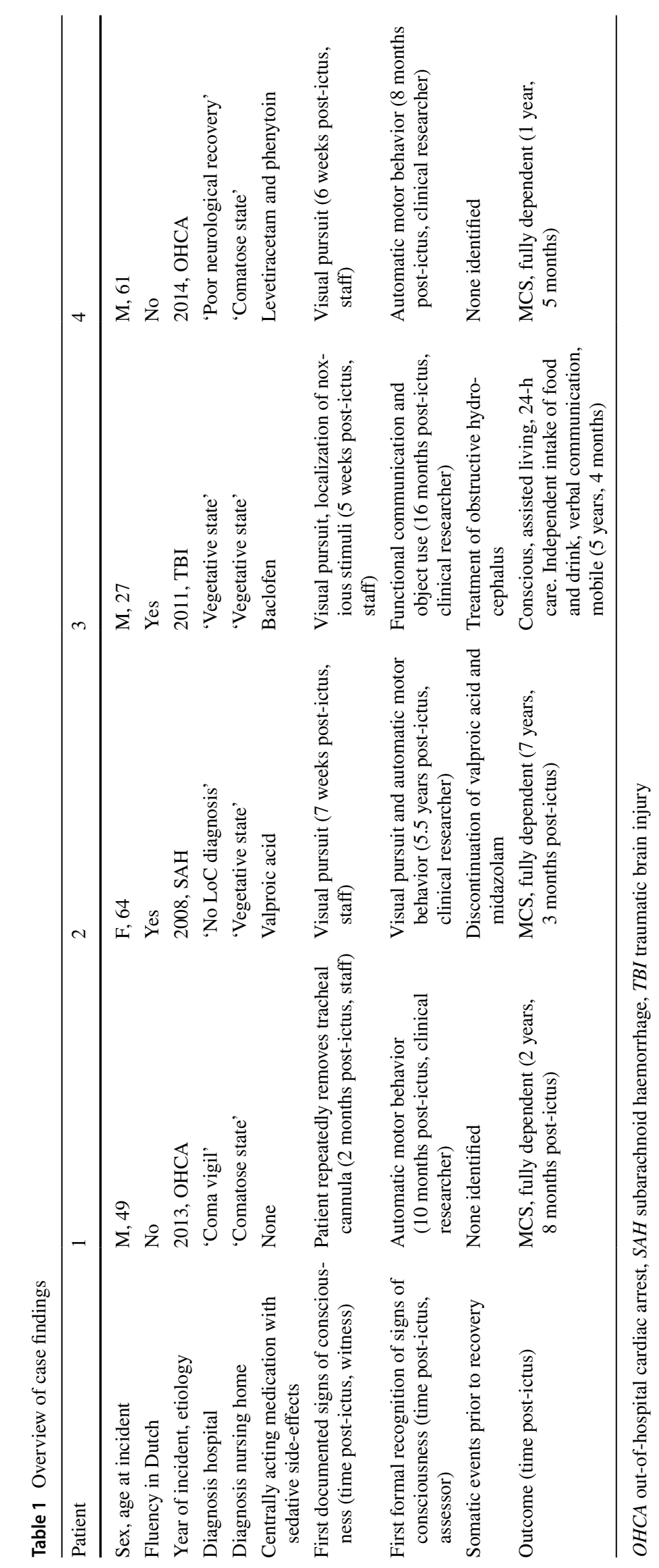


state'. When his reaction pattern deteriorated at 5 months after the injury, all paramedic therapies were discontinued. During research assessment only reflexes were seen. Following transfer to a regular nursing home closer to the patient's home-town, 8 months after the injury, a brain CT-scan made on the family's request demonstrated an obstructive hydrocephalus. An intraventricular drain was inserted. The patient recovered command following at 12 months, and functional communication at 16 months post-ictus corresponding to a conscious state. Three years on he lives in a supervised apartment. He eats, drinks and mobilizes independently and communicates verbally.

\section{Patient 4}

A 61-year-old male with limited Dutch proficiency survived an out-of-hospital cardiac arrest in 2014 in a state characterized by the cardiologist as 'poor neurological recovery'. Upon admission to a regular nursing home, his condition was described as a 'comatose state'. Six weeks after the incident the patient was noted to follow objects and people with his eyes; at 2 months post-ictus he showed nuanced facial expressions in relation to emotional context. The first formal CRS-R assessment at 5 months post-ictus by the study researcher elicited reflexes only. The family, despite instructing the patient in his native language, could not provoke conscious behavior either. At 8 month follow-up, the patient demonstrated reproducible automatic behavior consistent with MCS-. He was transferred to a specialized nursing home but showed no further improvement.

\section{Discussion}

On first sight, the stories in this case-series might be regarded as unexpected or 'miraculous' recoveries worthy of press attention [12]. On closer inspection, all patients showed signs of consciousness within the established prognostic timeframes. Rather than examples of remarkably late recovery of consciousness, these are cases of late discovery of consciousness, remarkable nonetheless [13]. Why was these patients' potential not recognized sooner?

First, there is the apparent absence of standardized behavioral assessment in daily practice, the inaccurate diagnostic terminology and the misinterpretation of conscious behavior. None of the patients' medical files or transfer letters mentioned validated scales for LoC determination, let alone repeated CRS-R assessments. Only one patient left the hospital with a formal, though outdated, diagnosis ('coma vigil'). Upon nursing home admission, two patients were incorrectly labeled as being in a 'comatose state', and the other two were considered 'vegetative' though both showed signs of consciousness. Those behaviors were, by all accounts, noticed but erroneously interpreted as 'unconscious reflexes'. When patient 3 subsequently stopped showing signs of consciousness, due to, as it turned out later, the development of hydrocephalus, no expert consultation was sought. Earlier referral for radiological evaluation might have detected this complication sooner.

The second factor concerns the various internal and external influences on patients' performances. Language barriers (patients 1 and 4), sedative drugs (patient 2) and patient 3's hydrocephalus are known diagnostic confounders [14-16], as are fluctuations in arousal and awareness [17]. Patient 1 , for example, showed only reflexive behavior during assessment while already capable of object manipulation (i.e. removal of his own tracheal cannula) and visual pursuit. Repeated CRS-R measurements might have detected such signs of consciousness earlier. Had it not been for the research project, however, some of the patients' recoveries might have not been discovered at all.

VS/UWS is a diagnosis per exclusionem: absence of proof is not the same as proof of absence. Only if a stimulus is perceived, processed and gives rise to specific motor behavior we know that someone is conscious [18]. Both input to and output of conscious brain processing may be hampered, thus masking what the patient is perceiving. The detection and, if possible, treatment of epilepsy, low arousal due to sedatives or metabolic impairments, dysphasia, motor impairment, sensory deficit, neglect, attention fluctuations, hydrocephalus, and compensation of language barriers should be regarded as prerequisites for a diagnosis of VS/UWS. On top of that, a formal diagnosis of VS/UWS or MCS warrants a minimum of 5 standardized clinical assessments at different timepoints within a short time interval (e.g. 14 days) [19].

In view of the low prevalence of disorders of consciousness in general [20], and limited specialized care for these conditions even in high-income nations such as the Netherlands [10], it is unreasonable to expect every clinician to be able to discriminate between VS/UWS and MCS. Underestimation of a patient's awareness, however, may lead to erroneous prognostication, therapeutic nihilism, inadequate clinical and pain management and misinformed end-of-life decisions. Delayed recognition of recovery of consciousness, particularly in long-term care, should also be taken into account during the continuing revision of the prognosis of VS/UWS. A recent publication on late emergence from VS/UWS hypothesized that recovery taking place between formal LoC assessments may go unnoticed [21]. Our caseseries proves this to be true. The actual moment of recovery of consciousness can precede its formal recognition by years. Still, this study cannot be seen as proof that late recovery is non-existent.

This study is of modest size and of only partially prospective nature. It is likely that a scientific or clinical context with structural CRS-R based evaluations in place for all 
patients with prolonged disorders of consciousness would identify late recognition of recovery more often. A mobile team, providing expert-level and evidence-based diagnostic assessment on-site and educating care professionals and families, could minimize future misdiagnoses, diagnostic delays and both scientific and public misconceptions about 'miracle recoveries'. Bringing the expert to the patient, instead of vice versa, takes away the practical challenges of a clinical transfer, as well as the reduced arousal that often becomes apparent after a patient in VS/UWS reaches hospital. Such a relatively simple innovation would bring patients with prolonged disorders of consciousness closer towards the care and attention they deserve.

Acknowledgements We thank the patients, families and our colleagues for their cooperation. This study was funded by the Stichting Beroepsopleiding Huisartsen.

Author contributions Willemijn van Erp contributed to the conception and design of the study, to the acquisition and analysis of data and drafted a significant proportion of the manuscript. Anoek Aben contributed to the acquisition and analysis of data and drafted a significant proportion of the manuscript. Jan Lavrijsen contributed to the conception and design of the study, to the acquisition and analysis of data and drafted a significant proportion of the manuscript. Pieter Vos drafted a significant proportion of the manuscript. Steven Laureys drafted a significant proportion of the manuscript. Raymond Koopmans drafted a significant proportion of the manuscript.

\section{Compliance with ethical standards}

Conflicts of interest The authors declare that they have no conflict of interest.

Ethical standards All procedures performed in studies involving human participants were in accordance with the ethical standards of the institutional and/or national research committee and with the 1964 Helsinki declaration and its later amendments or comparable ethical standards.

Informed consent Informed consent was obtained from the representatives of all individual participants included in the study.

Open Access This article is distributed under the terms of the Creative Commons Attribution 4.0 International License (http://creativeco mmons.org/licenses/by/4.0/), which permits unrestricted use, distribution, and reproduction in any medium, provided you give appropriate credit to the original author(s) and the source, provide a link to the Creative Commons license, and indicate if changes were made.

\section{References}

1. Laureys S, Celesia GG, Cohadon F, Lavrijsen J, Leon-Carrrion J, Sannita WG et al (2010) Unresponsive wakefulness syndrome: a new name for the vegetative state or apallic syndrome. BMC Med $8(1): 68$

2. Jennett B, Plum F (1972) Persistent vegetative state after brain damage. A syndrome in search of a name. Lancet 1(7753):734-737
3. The Multi-Society Task Force on PVS (1994) Medical aspects of the persistent vegetative state (1). N Engl J Med 330:1499-1508

4. Estraneo A, Moretta P, Loreto V, Lanzillo B, Santoro L, Trojano L (2010) Late recovery after traumatic, anoxic, or hemorrhagic long-lasting vegetative state. Neurology 75(3):239-245

5. Giacino JT, Katz DI, Schiff ND, Whyte J, Ashman EJ, Ashwal $S$ et al (2018) Practice guideline update recommendations summary: Disorders of consciousness: report of the Guideline Development, Dissemination, and Implementation Subcommittee of the American Academy of Neurology; the American Congress of Rehabilitation Medicine; and the National Institute on Disability, Independent Living, and Rehabilitation Research. Neurology 8(0 000000000005926):0000000000005926

6. Giacino JT, Ashwal S, Childs NL, Cranford R, Jennett B, Katz DI et al (2002) The minimally conscious state: definition and diagnostic criteria. Neurology 58:349-353

7. Laureys S, Perrin F, Faymonville ME, Schnakers C, Boly M, Bartsch V et al (2004) Cerebral processing in the minimally conscious state. Neurology 63(5):916-918

8. Laureys S, Owen AM, Schiff ND (2004) Brain function in coma, vegetative state, and related disorders. Lancet Neurol 3:537-546

9. Bruno MA, Vanhaudenhuyse A, Thibaut A, Moonen G, Laureys S (2011) From unresponsive wakefulness to minimally conscious PLUS and functional locked-in syndromes: recent advances in our understanding of disorders of consciousness. J Neurol 258(7):1373-1384

10. van Erp WS, Lavrijsen JC, Vos PE, Bor H, Laureys S, Koopmans RT (2015) The vegetative state: prevalence, misdiagnosis, and treatment limitations. J Am Med Dir Assoc 16(1):85e9-89e14

11. Giacino JT, Kalmar K, Whyte J (2004) The JFK Coma Recovery Scale-Revised: measurement characteristics and diagnostic utility. Arch Phys Med Rehabil 85(12):2020-2029

12. Wijdicks EF, Wijdicks MF (2006) Coverage of coma in headlines of US newspapers from 2001 through 2005. Mayo Clin Proc 81(10):1332-1336. https://doi.org/10.4065/81.10.1332

13. Wijdicks EF (2006) Minimally conscious state vs. persistent vegetative state: the case of Terry (Wallis) vs. the case of Terri (Schiavo). Mayo Clin Proc 81(9):1155-1158

14. Pickard JD, Coleman MR, Czosnyka M (2005) Hydrocephalus, ventriculomegaly and the vegetative state: a review. Neuropsychol Rehabil 15(3-4):224-236

15. Strens L, Mazibrada G, Duncan J, Greenwood R (2004) Misdiagnosing the vegetative state after severe brain injury: the influence of medication. Brain injury [BI] 18(2):213-218

16. Majerus S, Gill-Thwaites H, Andrews K, Laureys S (2005) Behavioral evaluation of consciousness in severe brain damage. Prog Brain Res 150:397-413

17. Piarulli A, Bergamasco M, Thibaut A, Cologan V, Gosseries O, Laureys S (2016) EEG ultradian rhythmicity differences in disorders of consciousness during wakefulness. J Neurol 263(9):17461760. https://doi.org/10.1007/s00415-016-8196-y (Epub 2016 Jun 13)

18. Giacino JT, Schnakers C, Rodriguez-Moreno D, Kalmar K, Schiff N, Hirsch J (2009) Behavioral assessment in patients with disorders of consciousness: gold standard or fool's gold3. Progress Brain Res. 177:33-48. https://doi.org/10.1016/S0079 -6123(09)17704-X

19. Wannez S, Heine L, Thonnard M, Gosseries O, Laureys S (2017) The repetition of behavioral assessments in diagnosis of disorders of consciousness. Ann Neurol 81(6):883-889. https://doi. org/10.1002/ana.24962

20. van Erp WS, Lavrijsen JC, van de Laar FA, Vos PE, Laureys S, Koopmans RT (2014) The vegetative state/unresponsive wakefulness syndrome: a systematic review of prevalence studies. Eur J Neurol 21(11):1361-1368 
21. Yelden K, Duport S, James LM, Kempny A, Farmer SF, Leff AP et al (2018) Late recovery of awareness in prolonged disorders of consciousness -a cross-sectional cohort study. Disabil Rehabil
40(20):2433-2438. https://doi.org/10.1080/09638288.2017.13392 09 (Epub 2017 Jun 21) 